\title{
Histological characteristics of periprosthetic tissue around metal on metal hip prostheses
}

\begin{abstract}
Background: The most noteworthy advantage of metal-on-metal (MoM) total hip prostheses is low were rate and avoidance of polyethylene (PE) particles. The aim of the present study was to evaluate the histological characteristics of the tissue around MoM THR and to determine if perivascular lymphoid aggregates are characteristic findings in periprosthetic tissue around MoM prostheses.
\end{abstract}

\begin{abstract}
Material and methods: Tissue samples from 26 revised THRs with MoM articulation and 36 samples from two control groups: metal on polyethylene (MoPE) and ceramic on polyethylene (AloPE) were analysed. Histopathological and immunohistochemical analyses of periprosthetic tissue samples were performed in order to determine general cellular features. Semi-quantitave rating method to classify the numbers of macrophages, giant cells, lymphocytes, as well as extend of necrosis was used. In the second part of the study tissue form 544consecutive THRs were analysed and cases with Oxford ALVAL scoring system $2+$ and $3+$ were selected regardless the type of prosthesis.
\end{abstract}

Results: The retrieved periprosthetic tissue showed granulomtous tissue with varying numbers of macrophages with different cytoplasmatic amount of metal particles. The characteristic histological features in periprosthetic tissue from MoM THRs were diffuse lymphocytes in $16(62 \%)$ of cases and perivascular lymphocytic cuffs in $15(58 \%)$ cases. In comparison with tissue from control groups the lymphocytic infiltration in tissue around MoM THRs was much more intense and the presence of foreign body type giant cells was much less marked. In second part of the study we found large perivascular lymphoid aggregates also in 8cases of non-MoM THR cases.

Conclusion: Extensive lymphocytic infiltration in the periprosthetic tissue around MoM THR is predominant histological pattern; however, perivascular lymphoid aggregates are not exclusive characteristic in tissue around MoM prostheses.
Volume 3 Issue I - 2019

\author{
Andrej Cör \\ Orthopedic Hospital Valdoltra, Slovenia
}

Correspondence: Andrej Cör, Orthopedic Hospital Valdoltra, Jadranska 3I,Ankaran, Slovenia, Tel +386 56696 393, Email vlulune@googlemail.com

Received: January II, 2019 | Published: February 25, 2019

\section{Introduction}

The ultimate challenge for orthopedic surgeons who implant hip prosthesis is providing long-term function of the joint without pain. The most common cause of total hip arthroplasty failure is aseptic loosening mostly initiated by wear particles. ${ }^{1}$ The current paradigm explains aseptic loosening as an inflammatory response to the wear debris particles produced by prosthetic implants. Wear particles are phagocytosed by macrophages and induce a variety of cytokines that mediate inflammatory response leading to the recruitment and activation of osteoclasts, and eventual implant loosening. ${ }^{2}$ For over 40 years, the metal-on-polyethylene (MoPE) bearing has dominated the field of total hip replacement (THR). The second generation of metal on metal (MoM) THRs made of CoCrMo alloy was introduced into the market in early 1990s with the aim to eliminate the wear of polyethylene (PE) and therefore, to achieve better long-term clinical results compared to conventional MoPE THRs. MoM artificial hip prostheses showed an extremely low rate of wear of the articulation surfaces. In addition, metal particles that are produced are smaller than PE particles and hence, they may induce less tissue reaction. ${ }^{3-5}$ However, patients with M-M articulations have increased levels of $\mathrm{Co}$ and $\mathrm{Cr}$ in the serum and urine, and this has raised concerns about toxicity, mutagenesis and hypersensitivity. ${ }^{6}$ There are reports from orthopedic registers about increase complications and potential problems of early failure of MoM, often require revision surgery. ${ }^{7,8}$ Several studies reported soft tissue damages with adverse reaction to metal debris. ${ }^{9}$

The histological appearances of the periprosthetic tissues are related to the type, number and size of particles. There have been numerous reports relating the histological characteristics of the periprosthetic tissue around MoPE THR ${ }^{4,7}$ As compared with MoPE THRs, the extend of the granulomatous inflammatory reaction and the presence of foreign body type giant cells are less intense in MoM THRs, likely because of the lower numbers and overall smaller size of metal wear particles..$^{10}$ On the other side, the presence of perivascular lymphoid aggregates in tissue around failed joint prostheses has been described as a pattern of inflammation that is characteristic and largely limited to failed MoM hip implants. For such histological picture special name "aseptic lymphocyte-dominated vasculitis associated lesion (ALVAL) « has been introduced in 2005 by Willert et al (5). They suggested that histological findings are part of delayed-type hypersensitivity like reaction. Two defined methodologies for histological analysis of such lesions have emerged in the literature. In 2010 CampbellALVAL scoring system ${ }^{11}$ and more recently, the Oxford-ALVAL scoring system were introduce. ${ }^{12}$ Oxford ALVAL scoring system strictly assesses the amount of perivascular lymphoid aggregates. The aim of our study was to assess the histological characteristics of tissue, collected after the revision surgery after aseptic loosening of MoM THRs and to determine if perivascular lymphoid aggregates are characteristic findings in periprosthetic tissue of MoM THR.

\section{Material and methods}

In first part of the study tissues from twenty-six patients with MoM articulation were collected at revision operation because of aseptic loosening. The ace tabular component was a Bicon-Plus M-M cup (Plus Orthopedics GA, Rotkreuz, Switzerland). An RCH-1000 Chirulen ultrahigh molecular weight polyethylene linear is interposed between the shell and the Sikomet SM21 Co-28Cr-6Mo low-carbon 
alloy articulating surface. A 28-mm diameter metal femoral ball head manufactured from Sikomet SM21 was used in all patients. Sikomet SM21 is a low carbon, forged, vacuum melted Co-28Cr-6Mo alloy exhibiting fine grain structure with an almost carbide free surface. From each patient tissue specimens from neo-capsule with additional material taken from ace tabular implant-bone interface were obtained. Institutional Ethic Review Board approved the study. Informed consent from each patient was obtained.

Tissues from two control groups of patients were also analysed. In the first control group tissue that had been retrieved from eighteen patients with cemented Chernley-Muller prostheses (Alivium, ZiMMer, Warsaw, Indiana USA) consisting of a curved monobloc femoral component made of CoCrMo alloy and a polyethylene cup (MoPE) were included. In the second control group there were tissue samples from eighteen patients with non-cemented TRHs free of cobalt and chromium, made of titanium-based alloy stem and $\mathrm{Al}_{2} \mathrm{O}_{3}$ femoral head and a polyethylene cup (AloPE). Revision operations were done due to aseptic loosening in control groups of patients as well. All primary implantations were performed due to osteoarthritis or femoral head necrosis. Patients with inflammatory disorders such as rheumatoid arthritis were excluded. Infection was ruled out by negative preoperative or intra operative cultures and none of the patients had neutrophilic granulocytes in obtained histological specimens.

Tissue samples were obtained intra operatively and immediately fixed in buffered $4 \%$ formalin, decalcified if necessary and embedded in paraffin. Five $\mathrm{cm}$ thick paraffin sections were stained with haematoxylin and eosin, Giemsa, Prussian blue and periodic acidSchiff methods. From each of the paraffin block additional sections were used for macrophages and lymphocytes immunohistochemical analysis. For immunohistochemistry $5 \mathrm{~cm}$ thick paraffin sections were dewaxed in xylene, rehidrated in alcohol and blocked for endogenous activity ( $3 \% \mathrm{H}_{2} \mathrm{O}_{2}$ in methanol and normal rabbit serum). Antigen retrieval was carried out by pressure-cooking in citrate buffer, $\mathrm{pH}$ 6.0. For macrophages analysis monoclonal anti human CD68 antibody (DAKO, Denmark) diluted 1: 100 and for lymphocytes T and B monoclonal human anti CD3 and CD20 antibody (DAKO, Denmark) diluted 1: 100 were used. After incubation with primary antibodies sections were washed and treated with biotinilated secondary antibody for 30min. Antigen visualisation was achieved by applying a standard streptavidin-biotin complex (ABC, DAKO, Denmark) for 30min followed by diaminobenzidin chromogen (DAB, Sigma Chemical Co, Germany) in $0.1 \% \mathrm{H}_{2} \mathrm{O}_{2}$ PBS solution. Sections were counterstained with haematoxylin. The specificity of antibodies used was checked with positive and negative control sections. Sections treated without the primary antibodies served as negative controls. For positive controls, human lymph node sections were processed in the same way as tissue from THRs.

Histopathological analysis of tissue samples was performed in order to determine general cellular features. We used a semi-quantitave rating method to classify the numbers of macrophages, giant cells, as well as extend of necrosis in ten high power microscopic fields. ${ }^{4}$ Polarized light was used to detect wear particles; birefringent particles were assumed to be ultra high molecular weight polyethylene and dense black particles, that have thin refractive edges when viewed with polarized light, were assumed to be metal particles. Lymphocytic infiltration was assessed by counting the individual cells of a diffuse infiltration and the number of perivascular lymphoid aggregates per field of view (Table 1). In cases where more than one tissue samples were available, the results were averaged. In the second part of our research we analysed histological slides from 544 consecutive patients with revision hip arthroplasty and cases with $2+$ or $3+$ according to Oxford-ALVAL scoring system were included in the study regardless the type of prosthesis. $2+$ or $3+$ are severe perivascular lymphoid aggregates with lymphocyte cuffing around vessels being more than five cells in thickness. For each patient clinical, microbiological and other histological characteristics (metalosis, macrophage infiltration and necrosis) as well as type of the prosthesis were determined.

Table I Grading of lymphocytic infiltration per field of 400-magnification for diffuse lymphocytes and per field of 40-magnification for perivascular lymphoid aggregates.

\begin{tabular}{lll}
\hline Grading & $\begin{array}{l}\text { Number of diffuse } \\
\text { lymphocytes }\end{array}$ & $\begin{array}{l}\text { Number of perivascular } \\
\text { lymphoid aggregates }\end{array}$ \\
\hline None $(0)$ & $\leq 10$ & 0 \\
Few $(1+)$ & $11-30$ & $1-2$ \\
Many $(2+)$ & $31-50$ & $3-6$ \\
Abundant $(3+)$ & $51-100$ & $7-10$ \\
Excessive $(4+)$ & $>100$ & $>10$ \\
\hline
\end{tabular}

\section{Results}

Twenty-six periprosthetic tissue specimens from patients after revised MoM hip prosthesis were analysed. There were 20 female and 6 male patients; the mean age was 65years ranging from 25 to 78 years. The median survival time (time in situ) of the implant was 77.4 months ranging from 10 to 136 months. Two additional groups of patients were also analysed. In MoPE group there were eighteen patients (11 female and 7 males, the mean age was 65 years) with median survival time of the implant 172 months. In AloPE group also eighteen patients (10 female and 8 males, the mean age was 77 years) with median survival time of the implant 262.5 months were included. The periprosthetic tissues from all patients with revised MoM implant showed a variety of appearance with mild foreign-body reaction to wear particles with various numbers of macrophages, fibroblasts, and blood vessels as well as zones of necrosis. In $8(31 \%)$ cases, histiocytes showed "slateblue cytoplasmic appearance according to Doorn et al., ${ }^{4}$ (Figure 1A). $15(58 \%)$ cases showed a few isolated black specks in the cytoplasm of the macrophages. "Jet-black" colour with abundant metallic debris was found in only $3(11 \%)$ neo-capsule tissue samples (Figure 1B). Among the macrophages extensive diffusely distributed lymphocytic infiltration were observed together with perivascular lymphoid aggregates. Many $(2+)$ or more diffusely distributed lymphocytes were found in $16(61 \%)$ cases of MoM implants (Figure 2A). In 15 $(58 \%)$ cases in intermediate vascular layer the lymphoid aggregates (Figure 2B) were found. Immunohistochemical analysis revealed that most tissue-infiltrating lymphocytes in the periprosthetic tissue expressed the CD3 marker and therefore could be identified as T-cells

In the tissues around MoPE THRs histological examination shows foreign-body reactions with extensive phagocytic granulomas contained sheets of macrophages and giant cells with engulfed polyethylene and metal particles. Using polarized light, a bluishwhite tissue brief ringenecy was seen in highly cellular areas, which probably corresponds to widespread submicron PE particles. In 6cases "slate blue" macrophages predominated but in 2 cases larger black particles were found in cytoplasm of macrophages. Immunohistochemical analysis demonstrated that mostly CD68positive macrophages and giant cells were seen (Figure 3). In three 
cases many $(2+)$ diffuse lymphocytes and in two cases perivascular lymphoid aggregate were found. In the tissue from control group II (AloPE) granulomatous reaction with macrophages and giant cells were found, but there was fewer giant cells with PE particles than in MoPE group. In 5cases "slate blue" macrophages predominate and in three cases black particles were seen in the cytoplasm of macrophages. Few $(1+)$ diffuse lymphocytes in 4 cases and many $(2+)$ lymphocytes in one case were found. Besides that, in one case with $2+$ diffuse lymphocytes we found also perivasculary lymphoid aggregates. In the second part of our study where we analysed histological slides from 544 patients obtained at the revision hip arthroplasties retrospectively and cases with $2+$ or $3+$ according to Oxford-ALVAL scoring system were included. We found 17 cases where MoM, and three additional cases were modular. In four cases, infection was microbiologically proven without histological criteria. However, in six of MoPe and two AloPe we also found perivascular lymphoid aggregates. Some of the lymphoid aggregates were quite large. No correlation between Oxford-ALVAL scoring system and other histological characteristics of periprosthetic tissue were found

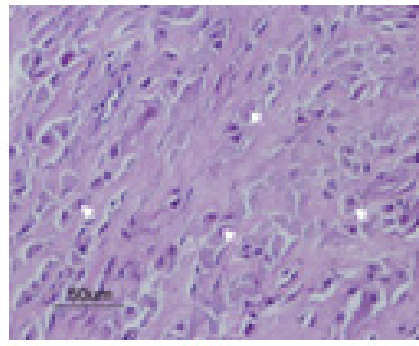

A

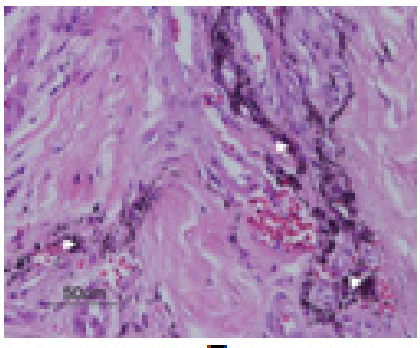

B
Figure I A, Macrophages with "slate blue" cytoplasm and B, macrophages filled up with black metal particles.

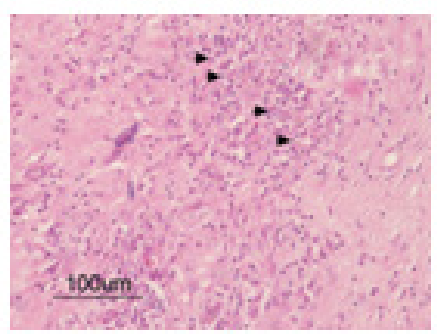

A

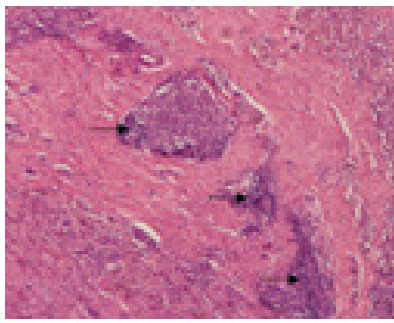

B
Figure 2 Photomcrograph demonstrating diffuse lymphocyte infiltration A) and lymphoid aggregates $(B)$ in the periprosthetic tissue around MoMTHRs.

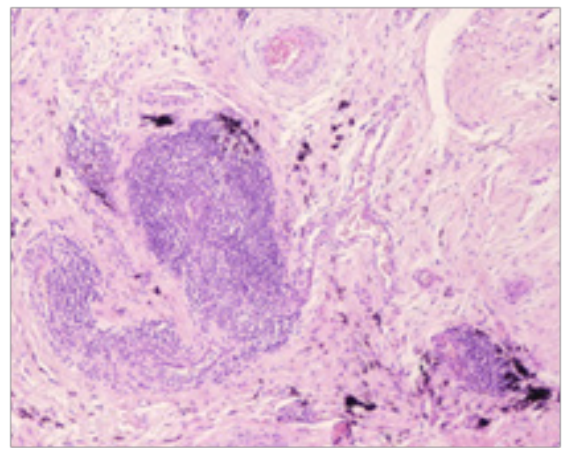

Figure 3 Large perivascular lymphoid aggregate $(3+$ according to Oxford ALVAL scoring system) from tissue around MoPE THR.

\section{Discussion}

The pathogenesis of loosening of joint replacement continues to be major focuses of research in orthopedics and many studies have found that polyethylene were particles largely contribute to that process. The second generation of MoM bearings was introduced with great optimism as an improvement on other types of bearings. Two decades ago, large percentages of THR performed used MoM bearing surfaces, however, in last few years there has been major decline in the use of this type of hip prostheses. The survivorship of MoM was fond to be lower in comparison to other types of bearing surfaces. $^{7}$ The particles produced on MoM prostheses are smaller than PE particles; however, more particles are produced by MoM as compared with MoPE bearings. ${ }^{13}$ The size of metal particles reported by scanning electron microscopy studies ranges from 0.1 to $5 \mathrm{~cm}$ and suggested that large metallic particles observed with light microscopy were agglomerates of the smaller particles. ${ }^{4}$ Several authors have implicated metal wear particles as a factor in the loosening of some MoM implants. In our study, there was only small amount of histologically visible metal particles and foreign-body reaction was relatively mild. In $31 \%$ of cases macrophages showed just "slateblue cytoplasmic appearance indicative of small (submicron) metal particle sizes. $58 \%$ of cases showed a few isolated black specks in the cytoplasm of the macrophages and only in three cases abundant debris with jet-black macrophages were presented. Our findings are in agreement with the results reported by Ebramzadeh et al., ${ }^{10}$ where they did not find any correlation between ALVAL scores and metalosis. Unlike polyethylene, $\mathrm{Co}-\mathrm{Cr}$ particles are not inert ${ }^{14}$ and have the potential to release metal ions. ${ }^{15}$ Patients with MoM articulations have increased levels of $\mathrm{Co}$ and $\mathrm{Cr}$ in the serum and urine, and this has raised concerns about toxicity, mutagenesis and hypersensitivity. ${ }^{6} \mathrm{At}$ this stage there is no epidemiological evidence to suggest that the risk of carcinogenesis is anything more than theoretical. ${ }^{16}$ However, many studies have shown increased lymphocytic infiltration in periprosthetic tissue samples from M-M revision ${ }^{15,17-19}$

Willert et al., ${ }^{5}$ first described an unusual perivascular lymphocytic infiltrate like that found in association with type-IV hypersensitivity. They characterized those changes by vasculitis with perivascular and intramural lymphocytic infiltration of the postcapilar venules, swelling of the vascular endothelium, recurrent localized bleeding and necrosis. They termed those histological changes as aseptic lymphocyte-dominated vasculitis associated lesion (ALVAL). Bauer et al. ${ }^{20}$ found perivascular lymphocytic infiltrates in 10 of $13(76.9 \%)$ cases, Davis et al., ${ }^{19}$ reported lymphocytic cuffs in 17 out of 25 $(68 \%)$ cases and Willert et al., ${ }^{5}$ found many, abundant or excessive perivascular lymphocytic infiltrates in 17 of $19(89 \%)$ cases of revised MoM THRs. Witzleb et al., ${ }^{18}$ reported a distinct lymphocytic infiltration in all cases with in situ times of more than 7 months.

Concerns about metal hypersensitivity in implants are not new and were well reviewed in 2001 by Hallab et al., ${ }^{21}$ Immunologically, metallic ions can serve as haptens and elicit a type IV hypersensitivity reaction. Delayed-type hypersensitivity is characterized by antigen activation of sensitized T-lymphocytes releasing various cytokines that result in the recruitment and activation of macrophages. ${ }^{17}$ Chronically loose implants with their associated metallic debris may sensitize persons who were previously without sensitivity. The most common metal sensitizer in human is nickel, followed by cobalt and chromium. However, significant immunologic reactions, including dermatitis and eczema, have not been widely reported after THRs. In our study, 
we observed many $(2+)$ or more diffuse lymphocytic infiltration and perivascular aggregates in 16 and 15 of twenty-six cases, respectively. Although we observed some diffuse lymphocytes in the tissue samples from control groups, but they were less frequent than in the MoM group. However, we found in eight cases perivascular lymphocytic cuffs also in both control groups. That is why we performed second part of the study, to find out if we could find large $(2+$ or $3+)$ lymphoid aggregates according to Oxford ALVAL scoring system also in periprosthetic tissue of non-MoM THR. We found large perivascular lymphoid aggregates in 8 non-MoM cases. The prevalence of lymphocytic infiltration in non-MoM was described in several studies. Willert et al., ${ }^{5}$ described lymphocyte infiltrates in 4out of $11 \mathrm{MoPE}$ control group cases. Von Domarus et al., ${ }^{22}$ found 4cases with more than 300lymphocytes T per high-power field in 26non-MoM analyzed cases. $\mathrm{Ng}$ et al., ${ }^{23}$ reported a high frequency of perivascular lymphoid aggregates in periprosthetic tissue from revised total knee prostheses. One of the possible reasons is allergy to bone cement components. Bircher et al., ${ }^{24}$ described that components of bone cement, such as benzoyl peroxide, may cause allergic complications.

\section{Conclusion}

Extensive lymphocytic infiltration in the periprosthetic tissue around MoM THR is predominant histological pattern; however, perivascular lymphoid aggregates are not exclusive characteristics in tissue around MoM prostheses. The presence and relevance of these lymphoid aggregates in non-metal on metal arthroplasty is unknown. Each case with histologically proven ALVAL morphological picture must be considered separately to determine the cause of such histological picture.

\section{Acknowledgments}

None.

\section{Conflicts of interest}

Author declares that there is no conflict of interest.

\section{References}

1. Amstutz HC, Campbell P, Kossovsky N, et al. Mechanism and clinical significance of wear debris-induced osteolysis. Clin Orthop. 1992;(276):7-18.

2. Campbell PA, Wang M, Amstutz HC, et al. Positive cytokine production in failed metal-on-metal total hip replacements. Acta Orthop Scand. 2002;73(5):506-512.

3. Wagner M, Wagner H. Medium-term results of a modern metal-onmetal system in total hip replacement. Clin Orthop. 2000;(379):123133.

4. Doorn PF, Campbell PA, Worrall J, et al. Metal wear particle characterization from metal on metal total hip replacements: transmission electron microscopy study of periprosthetic tissues and isolated particles. J Biomed Mater Res. 1998;42(1):103-111.

5. Willert HG, Buchhorn GH, Fayyazi A, et al. Metal-on-metal bearings and hypersensitivity in patients with artificial hip joints. A clinical and histomorphological study. J Bone Joint Surg Am. 200;87(1):28-36.

6. Milosev I, Pisot V, Campbell P. Serum levels of cobalt and chromium in patients with Sikomet metal-metal total hip replacements. J Orthop Res Off Publ Orthop Res Soc. 2005;23(3):526-535.
7. Milosev I, Trebse R, Kovac S, et al. Survivorship and retrieval analysis of Sikomet metal-on-metal total hip replacements at a mean of seven years. J Bone Joint Surg Am. 2006;88(6):1173-1182.

8. Drummond J, Tran P, Fary C. Metal-on-Metal Hip Arthroplasty: A Review of Adverse Reactions and Patient Management. J Funct Biomater. 2015;26;6(3):486-499.

9. Matharu GS, Eskelinen A, Judge A, et al. Revision surgery of metal-onmetal hip arthroplasties for adverse reactions to metal debris: A clinical update. Acta Orthop. 2018;89(3):278-288.

10. Ebramzadeh E, Campbell P, Tan TL, et al. Can wear explain the histological variation around metal-on-metal total hips? Clin Orthop. 2015;473(2):487-94.

11. Campbell P, Ebramzadeh E, Nelson S, et al. Histological Features of Pseudotumor-like Tissues From Metal-on-Metal Hips. Clin Orthop Relat Res. 2010;468(9):2321-2327.

12. Grammatopoulos G, Pandit H, Kamali A, et al. The Correlation of Wear with Histological Features After Failed Hip Resurfacing Arthroplasty. $J$ Bone Jt Surg-Am. 2013;95(12):e81.

13. Firkins PJ, Tipper JL, Saadatzadeh MR, et al. Quantitative analysis of wear and wear debris from metal-on-metal hip prostheses tested in a physiological hip joint simulator. Biomed Mater Eng. 2001;11(2):143157.

14. Brown C, Fisher J, Ingham E. Biological effects of clinically relevant wear particles from metal-on-metal hip prostheses. Proc Inst Mech Eng [H]. 2006;220(2):355-369.

15. Catelas I, Petit A, Zukor DJ, et al. TNF-alpha secretion and macrophage mortality induced by cobalt and chromium ions in vitro-qualitative analysis of apoptosis. Biomaterials. 2003;24(3):383-391.

16. Levašič V, Milošev I, Zadnik V. Risk of cancer after primary total hip replacement: The influence of bearings, cementation and the material of the stem. Acta Orthop. 2018;89(2):252.

17. Park YS, Chon CY, Kim H, et al. [Changes of ascites nitric oxide according to the treatment course in cirrhotic patients with spontaneous bacterial peritonitis]. Korean J Hepatol. 2004;10(3):207-215.

18. Witzleb WC, Hanisch U, Kolar N, et al. Neo-capsule tissue reactions in metal-on-metal hip arthroplasty. Acta Orthop. 2007;78(2):211-220.

19. Davies AP, Willert HG, Campbell PA, et al. An Unusual Lymphocytic Perivascular Infiltration in Tissues Around Contemporary Metal-onMetal Joint Replacements: J Bone Jt Surg. 2005;87(1):18-27.

20. Baur W, Honle W, Willert HG, et al. Pathologische Veronderungen im umgebenden Gewebe von revidierten Metall-Metall-Gleitpaarungen. Orthopede. 2005;34(3):225-233.

21. Hallab NJ, Anderson S, Stafford T, et al. Lymphocyte responses in patients with total hip arthroplasty. J Orthop Res Off Publ Orthop Res Soc. 2005;23(2):384-391.

22. Von Domarus C, Rosenberg JP, Rüther W, et al. Necrobiosis and T-lymphocyte infiltration in retrieved aseptically loosened metal-onpolyethylene arthroplasties. Acta Orthop. 2011;82(5):596-601.

23. Ng VY, Lombardi AV, Berend KR, et al. Perivascular lymphocytic infiltration is not limited to metal-on-metal bearings. Clin Orthop. 2011;469(2):523-529.

24. Bircher A, Friederich NF, Seelig W, et al. Allergic complications from orthopaedic joint implants: the role of delayed hypersensitivity to benzoyl peroxide in bone cement. Contact Dermatitis. 2012;66(1):20 26. 